\title{
Thermo-molecular orientation effects in fluids of dipolar dumbbells
}

\author{
Christopher D. Daub, ${ }^{1, *}$ Per-Olof Åstrand, ${ }^{1}$ and Fernando Bresme ${ }^{2,1}$ \\ ${ }^{1}$ Department of Chemistry, Norwegian University of Science \\ and Technology (NTNU), Trondheim, Norway NO-7491 \\ ${ }^{2}$ Department of Chemistry, Chemical Physics Section, \\ Imperial College London, SW7 2AZ, London, UK
}

(Dated: September 1, 2014)

\begin{abstract}
We use molecular dynamics simulations in applied thermal gradients to study thermomolecular orientation (TMO) of size-asymmetric dipolar dumbbells with different molecular dipole moments. We find that the direction of the TMO is the same as in apolar dumbbells of the same size, i.e. the smaller atom in the dumbbell tends to orient towards the colder temperature. The ratio of the electrical polarization to the magnitude of the thermal gradient does not vary much with the magnitude of the molecular dipole moment. We also investigate a novel second order TMO that persists even in size-symmetric dipolar dumbbells where molecules have a slight tendency to orient perpendicular to the gradient except very close to the hot region, where (anti-)parallel orientations are preferred. Finally, we investigate rotational correlation functions and characteristic rotational times in these systems in an attempt to model possible spectroscopic signatures of TMO in experiments. Although we cannot detect any difference in integrated rotational times between equilibrium simulations and simulations in a thermal gradient, more careful modelling of the anisotropic rotational dynamics in the thermal gradient may be more successful.
\end{abstract}

PACS numbers:

${ }^{*}$ Corresponding author; Electronic address: christopher.daub@ntnu.no 


\section{INTRODUCTION}

Thermo-molecular orientation (TMO) refers to a phenomenon observed in nonequilibrium molecular dynamics (NEMD) computer simulations of fluids in a strong thermal gradient. Driven by the heat flux, aspherical molecules will prefer to orient themselves in a particular way; for example, analogous with the Soret effect observed in binary mixtures [1], a molecule with a mass asymmetry will prefer to have the heavier end pointed in the direction of the lower temperature [2]. In the case of a size asymmetry, in the absence of other competing asymmetric effects the smaller part of the molecule will point towards the lower temperature $[2,3]$. If the molecular fluid consists of polar particles (eg. water), the TMO effect can lead to the production of significant electric fields which might conceivably be of more than just theoretical interest [4-7], eg. for conversion of waste heat into electrical power.

Work in our research group on TMO has spanned a range of systems, from non-polar dumbbell molecules $[2,3]$ to a range of both rigid and flexible water molecules [4-7] as well as continuing work on some more complex systems [8]. In this paper, we report on our findings regarding TMO in rigid, dipolar dumbbells consisting of positively and negatively charged Lennard-Jones spheres bound together. Studying this system provides a way of bridging the gap between our previous work on nonpolar dumbbells and more complex realistic systems such as water. Dipolar dumbbells are interesting models in their own right, as simple but realistic approximations to real molecules such as carbon monoxide, or ionic salts at high temperature and low density which tend to form dimers [9-12], and this study contributes to the body of work already existing on the behaviour of these systems [13-17]. In particular, we are unaware of any other computer simulation studies of size-asymmetric dipolar dumbbells (but see Ref. 18), although extensive work has been done on the size-asymmetric primitive model consisting of charged hard spheres [19-23].

One of the goals of our work is to achieve experimental confirmation of the TMO effect, heretofore only observed in computer simulations. Although we do not present experimental results in this work, the spectroscopic signatures of a change in orientational preferences ought to be reflected in the dipole moment time-correlation functions. A diatomic dumbbell represents just about the simplest system that interacts with light, and so we might hope to compute the impact of the thermal gradient on the dipolar fluctuations. To that end, we 
have computed rotational time-correlation functions of the molecular dipole moment. Our results point the way towards understanding what sorts of spectroscopic evidence we might observe in the optical Kerr effect (OKE) and/or dielectric spectrum of water or another polar liquid in a thermal gradient that could confirm a TMO effect.

The outline of the paper is as follows. In Section II we detail the potential models we use, the simulation methods and other theoretical material necessary for understanding the results. In Section III we describe the results of the work, including critical parameters, plots of the equations of state, heat flux and orientational profiles across the thermal gradient for all of the models we studied, and the analysis of the rotational correlation functions. Finally, in Section IV we arrive at some conclusions and indicate the future directions of our work in this area.

\section{BACKGROUND THEORY AND SIMULATION PROTOCOLS}

\section{A. Potential models}

Our starting point is the model used in Ref. 3. Briefly, we simulate fluids consisting of collections of pairs of connected Lennard-Jones spheres (ie. dumbbells) using classical molecular dynamics simulations. The spheres are either equally sized or can be size asymmetric with a size ratio $\sigma_{+} / \sigma_{-}=2$, with the mean particle size $\sigma_{\mathrm{LJ}}^{*}=\sigma_{+}^{*}+\sigma_{-}^{*}=1$ in all cases. There are two main differences between the models considered previously [2, 3] and those we study here. First, we have added positive and negative charges to the spheres in order to produce dipolar dumbbells. In the size-asymmetric systems, we arbitrarily chose to place the positive charge on the larger atom. The opposite choice would simply have led to an electric polarization in the opposite direction. The charge magnitudes considered are $q / e= \pm 0.21,0.5$ and 1.0, with the equivalent resultant molecular dipole moment given in Table I. In particular, $q / e= \pm 0.21$ was chosen because it represents the charge at which the Lennard-Jones energy scale given by the well depth $\epsilon_{\mathrm{LJ}}$ is equal to the Coulombic energy scale given by $4 \pi \epsilon_{0} \sigma_{\mathrm{LJ}} / q^{2}$. Second, instead of having harmonic bonds between the two halves of the dumbbell we have joined them with rigid bonds. The bond length was chosen to match that of carbon monoxide, $d=0.128 \mathrm{~nm}$ or $d^{*}=d / \sigma_{\mathrm{LJ}}=0.391$ [24]. All of the potential parameters are detailed in Table I. Note that we have used reduced Lennard-Jones units in all 
TABLE I: Summary of potential models, systems studied and their critical parameters. $\epsilon_{\mathrm{LJ}}^{*}=1.0$ and $\sigma_{\mathrm{LJ}}^{*}=\left(\sigma_{+}^{*}+\sigma_{-}^{*}\right) / 2=1$ in all cases. The bond length separating each atom $d^{*}=0.391$ and the atomic masses $m_{+,-}^{*}=1$ in all cases. The real dipole moment magnitude $|\vec{\mu}|$ is given for a carbon monoxide model with $\sigma_{\mathrm{LJ}}=0.32717 \mathrm{~nm}$ [24]. Uncertainties in the critical parameters are estimates based on the results of many simulations in the critical region (see text for more information).

\begin{tabular}{c|c|c|c|c|c} 
System & $q / e$ & $|\vec{\mu}| / D$ & $\sigma_{+} / \sigma_{-}$ & $T_{c}^{*}$ & $\rho_{c}^{*}$ \\
\hline 1 & 0 & 0 & 1.0 & $2.43 \pm 0.02$ & $0.22 \pm 0.02$ \\
2 & 0 & 0 & 2.0 & $1.52 \pm 0.02$ & $0.15 \pm 0.02$ \\
3 & \pm 0.21 & 1.29 & 1.0 & $3.03 \pm 0.05$ & $0.24 \pm 0.03$ \\
4 & \pm 0.21 & 1.29 & 2.0 & $2.00 \pm 0.03$ & $0.13 \pm 0.02$ \\
5 & \pm 0.5 & 3.07 & 1.0 & $7.75 \pm 0.10$ & $0.18 \pm 0.03$ \\
6 & \pm 0.5 & 3.07 & 2.0 & $5.35 \pm 0.10$ & $0.11 \pm 0.02$ \\
7 & \pm 1.0 & 6.15 & 1.0 & $30.1 \pm 0.2$ & $0.16 \pm 0.02$ \\
8 & \pm 1.0 & 6.15 & 2.0 & $17.0 \pm 0.5$ & $0.11 \pm 0.02$ \\
\hline
\end{tabular}

simulations. Real units can be recovered using the parameters defined for carbon monoxide in Ref. 24, i.e. $\epsilon_{\mathrm{LJ}}=0.352 \mathrm{~kJ} / \mathrm{mol}, \sigma_{\mathrm{LJ}}=0.32717 \mathrm{~nm}$, and $m_{\mathrm{LJ}}=m_{+}+m_{-}=28 \mathrm{~g} / \mathrm{mol}$.

\section{B. Simulation methods}

All simulations were performed with one of two recent versions (either Jan 11, 2013 or Aug 16, 2013) of the LAMMPS simulation package [25]. Lennard-Jones interactions were computed within a cutoff of $2.5 \sigma_{\mathrm{LJ}}^{*}$. Coulombic interactions were computed with the Wolf method [26, 27], using a damping parameter $\alpha^{*}=\alpha / \sigma_{\mathrm{LJ}}^{*}=0.3$ and a cutoff of $3.5 \sigma_{\mathrm{LJ}}^{*}$. Similar parameters have been found to be accurate for simulations of water in thermal gradients [28] and we have verified that these parameters give potential energies in agreement with Ewald summation for the dipolar dumbbells; as an example, for system 7 in Table I at $T^{*}=T_{\mathrm{c}}^{*}$, $\rho^{*}=2.5 \rho_{\mathrm{c}}^{*}$ we obtained an average pair interaction energy per molecule $\left\langle U_{\text {pair }}^{*} / N\right\rangle=-99.6$ with the Ewald sum and -98.0 with the Wolf method, with a standard deviation in the instantaneous quantities of 0.6. The timestep in all production runs was $1.47 \mathrm{fs}(=0.0005$ in reduced units), with smaller timesteps used in some cases during initial equilibration (see 
below). Bonds were held rigid with the SHAKE algorithm [29].

\section{Determination of the critical point}

Before we can implement the temperature gradient we must first determine the critical point for the liquid-vapour transition of all of our models. We take an initial configuration of $N=1954$ dumbbells in an orthorhombic simulation cell of dimensions $L^{*} \times L^{*} \times 3 L^{*}$, where the reduced length $L^{*}=11$. This box can then be deformed gradually while performing NVT simulations to obtain a desired initial density $\rho^{*}$. Following an equilibration period at least $2 \mathrm{~ns}$ long at a given temperature, the configuration was recorded at intervals of 1.47 ps (1000 timesteps) for another $10^{7}$ timesteps. The resulting trajectory was then analyzed, and the local density in sub-volumes of the simulation cell was computed and built up into a histogram.

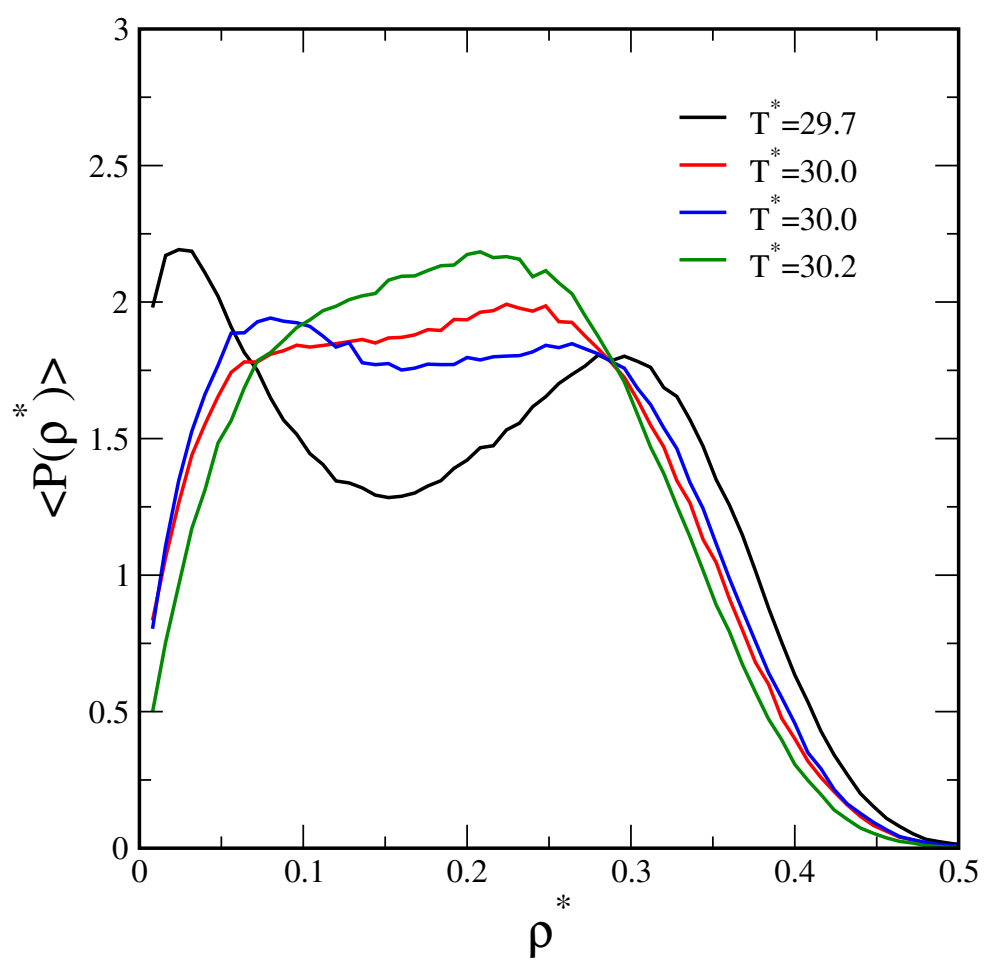

FIG. 1: Plots of the density histograms in the critical region of the size-symmetric model with $q / e= \pm 1$ (system 7 in Table I). The average density $\rho_{\mathrm{av}}^{*}=0.193\left(N=1954, L^{*}=15\right) .\left\langle P\left(\rho^{*}\right)\right\rangle$ represents the count in each trajectory snapshot of the local density in a total of $81(3 \times 3 \times 9)$ cubic subvolumes of the total simulation cell, averaged over 20000 snapshots. 
Examples of these histograms are shown in Figure 1 showing the critical region for one of our systems (system 7 in Table I). Above the critical temperature, the system is a homogeneous fluid and the density histogram has only a single peak. Below and within the spinodal region, the system phase separates, and the density histogram splits into two peaks with densities $\rho_{\mathrm{v}}$ and $\rho_{\mathrm{l}}$. By careful tuning of the temperature and total density to locate the temperature at which the two peaks merge into one, the critical point can be determined quite accurately. In particular the critical temperature can be determined to a precision of less than $1 \%$ relative uncertainty but the critical density is more uncertain, relating to how precisely $\rho_{\mathrm{v}}$ and $\rho_{\mathrm{l}}$ can be determined near coexistence as well as fluctuations in results from multiple runs at identical state points. The uncertainties listed in the critical parameters in Table I are conservative estimates based on these results, but cannot be said to correspond to any precise statistical uncertainty.

Our methodology neglects some important factors, and improved methods certainly exist for making a more accurate determination of the critical point in similar systems $[13,17,30]$. In particular, finite size effects can affect the location of the critical point[31]; however accounting for these effects would require multiple simulations with different system sizes, and a more involved analysis of the simulation data. For our purposes the simple method we have devised works reasonably well, and at least in the non-polar case gives results in good agreement with a more accurate method [3]; the differences in critical parameters we report here compared with Ref. 3 arise from issues with use of a Nosé-Hoover thermostat to equilibrate vibrational degrees of freedom (vide infra), and not from the method used to determine the critical parameters per se.

\section{NEMD simulations in a temperature gradient}

The method we use for generating a thermal gradient has been described elsewhere [3]. Briefly, we designate slabs of width $\sigma_{\mathrm{LJ}}$ at the edges of the simulation box $\left(3 L^{*} / 2<\right.$ $\left.|z|<3 L^{*} / 2-\sigma_{\mathrm{LJ}} / 2\right)$ as a region with a high temperature $T_{\text {hot }}$ and in the middle $\left(-\sigma_{\mathrm{LJ}} / 2<z<\sigma_{\mathrm{LJ}} / 2\right)$ as a region of low temperature $T_{\text {cold }}$, respectively. These regions are thermostatted using a Langevin thermostat [32], while the remainder of the simulation box obeys $N V E$ dynamics. The temperatures are chosen to give the desired magnitude of the thermal gradient $\nabla T^{*}=\left(T_{\text {hot }}^{*}-T_{\text {cold }}^{*}\right) /\left(3 L^{*} / 2\right)$, although it must be noted that the local 
gradient will differ significantly from this simple linear approximation [33]. The two halves of the simulation box from $0<z<3 L / 2$ and from $3 L / 2<z>3 L$ therefore experience a thermal gradient and subsequent heat flux equal and opposite to that in the other half of the cell in the direction of the $z$ axis. The temperature gradient points in the $-z$ direction in the left half of the simulation box, and in the $+z$ direction in the right half. The simulation box is always orthorhombic with dimensions $L^{*} \times L^{*} \times 3 L^{*}$.

The heat flux $J^{*}$ is obtained directly from the instantaneous per-atom stress tensor $\vec{S}_{i}$ and the kinetic and potential energies $E_{k, i}$ and $U_{i}$ of all $N_{a}$ atoms, according to $[28,34]$

$$
\vec{J}(V)=\frac{1}{V}\left[\sum_{i=1}^{N_{a} \in V}\left(E_{k, i}+U_{i}\right) \vec{v}_{i}-\vec{S}_{i} \vec{v}_{i}\right] .
$$

where $v_{i}$ is the instantaneous velocity of atom $i$. We compute the heat flux throughout the simulation cell as a function of $z$ in thin slabs each of subvolume $V$.

We first equilibrate the systems, starting from a lower temperature and reduced timestep and gradually increasing both while deforming the simulation box from its initial configuration until the desired average density is obtained. Because the initial configurations included

flexible bonds, and had a different density, the reduced timestep was necessary during initial equilibration to ensure that the SHAKE algorithm would not fail. This initial stage takes place over $2 \times 10^{6}$ timesteps with a maximum timestep of $1.47 / 5=0.294$ fs. Following this we equilibrate for another $1-2 \mathrm{~ns}$ with timestep 1.47 fs before we set up the thermal gradient. Then, we allow the system to reach steady state over the course of another $2-4$ ns before collecting data. Trajectory sampling for generating orientation profiles, equations of state and heat flux profiles took place at intervals of 1.47 ps over the course of at least $10 \mathrm{~ns}$ of simulation time.

\section{Rotational correlation functions}

In a sense, much of spectroscopy consists of measuring changes in the system dipole moment with time, and in many cases this can be related to the molecular transition dipole moment. For rigid molecules, measuring the average time required for a molecule to rotate will be related to its spectroscopic properties. To that end we calculate the rotational correlation functions of each molecular dipole $\vec{\mu}=\vec{r}_{+}-\vec{r}_{-}$of our systems, with $r_{+}$and $r_{-}$ being the positions of the positive and negative charge. In the case of the nonpolar molecules, 
this is just the vector joining the atoms - from small to large in the size-asymmetric case. The rotational correlation functions are given by [35]

$$
C_{n}(t)=\left\langle P_{n}\left[\vec{\mu}\left(t+t_{0}\right) \cdot \vec{\mu}\left(t_{0}\right) /|\vec{\mu}|^{2}\right]\right\rangle
$$

where $P_{n}[x]$ is the $n^{t h}$ order Legendre polynomial. $C_{2}(t)$ can be related to the Raman spectrum or the optical Kerr effect [35] and so this is what we focus on in this paper. To a rough approximation (assuming Debye type relaxation) the rotational correlation functions decay exponentially, and so it is feasible to simply extract a rotational correlation time $\tau_{n}$ from a fit of $C_{n}(t)=\exp \left(-t / \tau_{n}\right)$. At the same time, there is much debate on the proper way to model real molecular rotational correlation functions beyond the Debye approximation, for example as a sum of multiple exponentials or stretched exponentials [36-38]. In this work, we will simply obtain a numerically integrated correlation time,

$$
\tau_{n}=\int_{0}^{\infty} C_{n}(t) d t .
$$

We measure these rotational correlation times across the temperature gradient by subdividing the simulation cell into a number of slabs. It is worth considering whether a molecule is in a given slab if it is located there at either or both times $t=t_{0}$ and $t=t+t_{0}$. In the case of translational diffusion in the solvation shell of a protein [39] or hydrogen bond correlation functions near an interface [40], these choices have a large impact on the measured dynamics if the geometry is so restricted that a given molecule might leave the subvolume within the timescale of the dynamics. In our case, however, we found that the impact of the different geometric restrictions is negligible, owing to the fact that the rotational dynamics are considerably faster than translational diffusion. Due to these fast timescales, configurations were sampled more often for these calculations, every 20 time steps (29.4 fs) and collecting data for $3 \times 10^{5}$ time steps (441 ps).

\section{RESULTS}

The critical parameters we obtained are listed in Table I. As mentioned above, our

results for the apolar dumbbells differ significantly from those obtained previously [3] due to a subtle issue we have noticed in simulations of molecules with stiff yet flexible bonds. 
In some cases, a Nosé-Hoover thermostat [41, 42] or velocity scaling may be inadequate to properly equilibrate these vibrational degrees of freedom, leading to an apparent breakdown of equipartition and inaccurate temperature measurements. For example, in our previous work on apolar dumbbells we obtained a value of $T_{\mathrm{c}}^{*}=1.35$ for the size symmetric case, compared with our new value of $T_{\mathrm{c}}^{*}=1.53$. Because the vibrational degree of freedom was in effect too cold, the remaining degrees of freedom compensated by being too hot and so the apparent resulting $T_{\mathrm{c}}^{*}$ was much lower even though the effective temperature was the same. The same trend was observed in most of the rest of our systems; for instance with flexible bonds and a Nosé-Hoover thermostat we obtained $T_{\mathrm{c}}^{*}=4.7$ for system 5 , and $T_{\mathrm{c}}^{*}=5.35$ with bonds held rigid.

In this paper, we have avoided these problems by simulating molecules with rigid bonds, and using a Langevin thermostat [32] instead of velocity scaling to set up the thermal gradient. In fact, there is little difference in the critical parameters obtained for the systems with rigid or flexible bonds so long as the vibrational degree of freedom is adequately equilibrated (results not shown).

It is not surprising that the critical temperature rises with the charge magnitude, since much more potential energy is added into the system compared with the Lennard-Jones dumbbells. In fact, with analogous primitive model electrolytes one would expect $T_{\mathrm{c}} \propto$ $q^{2}$ [13]. The fact that the critical temperature increases less dramatically demonstrates that the Coulombic interaction is somewhat screened. As in the nonpolar dumbbells, the size-asymmetric systems have significantly lower critical parameters. This has also been noted in simulations of the size-asymmetric primitive model $[19,21]$. There is a weak trend showing a decrease in critical density with dipolar strength. This agrees with previous work on charged hard dumbbells where the dipole moment was increased by increasing the bond length separating the charges [14].

We then ran NEMD simulations in thermal gradients at state points corresponding to three different sets of average temperature $T_{\mathrm{av}}=\left(T_{\text {hot }}-T_{\text {cold }}\right) / 2$ and average number density $\rho_{\mathrm{av}}=N /\left(3 L^{3}\right):$ a) $T_{\mathrm{av}}=T_{\mathrm{c}}, \rho_{\mathrm{av}}=2.5 \rho_{\mathrm{c}}$, b) $T_{\mathrm{av}}=1.2 T_{\mathrm{c}}, \rho_{\mathrm{av}}=2 \rho_{\mathrm{c}}$, c) $T_{\mathrm{av}}=1.5 T_{\mathrm{c}}$, $\rho_{\mathrm{av}}=1.5 \rho_{\mathrm{c}}$. We also simulated two different system sizes, a larger system with $N=1954$ dumbbells and a smaller one with $N=770$. In total there are 6 NEMD simulations for each of our 8 potentials. All of the simulation conditions are summarized in Table II. In the apolar case, we used the same thermal gradient value as was used previously [3], i.e. 
$\nabla T^{*}=0.077$. The dipolar dumbbells were simulated in larger thermal gradients, reflecting the higher critical temperatures of these systems; the relative gradient $\nabla T^{*} / T_{\mathrm{c}}^{*}$ is of similar magnitude in all cases. At some state points with lower temperatures, it was necessary to reduce the magnitude of the gradient in order to avoid phase separation. 
TABLE II: Summary of NEMD simulation conditions. System identifiers in first column will be used in figure legends.

\begin{tabular}{|c|c|c|c|c|c|c|c|c|}
\hline System & $q / e$ & $\sigma_{+} / \sigma_{-}$ & $N$ & $\rho_{\mathrm{av}}^{*}$ & $L^{*}$ & $\nabla T^{*}$ & $T_{\mathrm{c}}^{*}$ & $T_{\mathrm{h}}^{*}$ \\
\hline $1 \mathrm{a}$ & 0 & 1.0 & 1954 & 0.55 & 10.58 & 0.077 & 1.819 & 3.041 \\
\hline $1 b$ & & & 770 & 0.55 & 7.76 & 0.077 & 1.982 & 2.878 \\
\hline $1 c$ & & & 1954 & 0.44 & 11.40 & 0.077 & 2.258 & 3.574 \\
\hline $1 d$ & & & 770 & 0.44 & 8.36 & 0.077 & 2.433 & 3.399 \\
\hline $1 \mathrm{e}$ & & & 1954 & 0.33 & 12.54 & 0.077 & 2.921 & 4.369 \\
\hline $1 \mathrm{f}$ & & & 770 & 0.33 & 9.20 & 0.077 & 3.114 & 4.176 \\
\hline $2 \mathrm{a}$ & 0 & 2.0 & 1954 & 0.375 & 12.02 & 0.077 & 0.826 & 2.214 \\
\hline $2 b$ & & & 770 & 0.375 & 8.81 & 0.077 & 1.011 & 2.029 \\
\hline $2 c$ & & & 1954 & 0.30 & 12.95 & 0.077 & 1.076 & 2.572 \\
\hline $2 \mathrm{~d}$ & & & 770 & 0.30 & 9.49 & 0.077 & 1.276 & 2.372 \\
\hline $2 \mathrm{e}$ & & & 1954 & 0.225 & 14.25 & 0.077 & 1.457 & 3.103 \\
\hline $2 f$ & & & 770 & 0.225 & 10.45 & 0.077 & 1.677 & 2.884 \\
\hline $3 a$ & \pm 0.21 & 1.0 & 1954 & 0.60 & 10.28 & 0.077 & 2.44 & 3.62 \\
\hline $3 b$ & & & 770 & 0.60 & 7.53 & 0.077 & 2.60 & 3.46 \\
\hline $3 c$ & & & 1954 & 0.48 & 11.07 & 0.077 & 3.00 & 4.28 \\
\hline $3 \mathrm{~d}$ & & & 770 & 0.48 & 8.12 & 0.077 & 3.17 & 4.11 \\
\hline $3 \mathrm{e}$ & & & 1954 & 0.36 & 12.19 & 0.077 & 3.85 & 5.25 \\
\hline $3 \mathrm{f}$ & & & 770 & 0.36 & 8.93 & 0.077 & 4.03 & 5.07 \\
\hline $4 a$ & \pm 0.21 & 2.0 & 1954 & 0.325 & 12.61 & 0.077 & 1.272 & 2.728 \\
\hline $4 \mathrm{~b}$ & & & 770 & 0.325 & 9.24 & 0.077 & 1.466 & 2.534 \\
\hline $4 \mathrm{c}$ & & & 1954 & 0.26 & 13.58 & 0.051 & 1.877 & 2.923 \\
\hline $4 \mathrm{~d}$ & & & 770 & 0.26 & 9.96 & 0.077 & 1.825 & 2.975 \\
\hline $4 \mathrm{e}$ & & & 1954 & 0.195 & 14.95 & 0.077 & 2.137 & 3.864 \\
\hline $4 \mathrm{f}$ & & & 770 & 0.195 & 10.96 & 0.077 & 2.367 & 3.633 \\
\hline $5 \mathrm{a}$ & \pm 0.5 & 1.0 & 1954 & 0.45 & 11.31 & 0.154 & 6.45 & 9.06 \\
\hline $5 b$ & & & 770 & 0.45 & 8.29 & 0.154 & 6.79 & 8.71 \\
\hline $5 c$ & & & 1954 & 0.36 & 12.19 & 0.154 & 7.89 & 10.71 \\
\hline $5 \mathrm{~d}$ & & & 770 & 0.36 & 8.93 & 0.154 & 8.27 & 10.33 \\
\hline $5 \mathrm{e}$ & & & 1954 & 0.27 & 13.41 & 0.154 & 10.08 & 13.17 \\
\hline 5 & & & 770 & 0.27 & 9.83 & 0.154 & 10.49 & 12.76 \\
\hline
\end{tabular}




\begin{tabular}{|c|c|c|c|c|c|c|c|c|}
\hline System & $q / e$ & $\sigma_{+} / \sigma_{-}$ & $N$ & $\rho_{\mathrm{av}}^{*}$ & $L^{*}$ & $\nabla T^{*}$ & $T_{\mathrm{c}}^{*}$ & $T_{\mathrm{h}}^{*}$ \\
\hline $6 \mathrm{a}$ & \pm 0.5 & 2.0 & 1954 & 0.275 & 13.33 & 0.1155 & 4.195 & 6.505 \\
\hline $6 \mathrm{~b}$ & & & 770 & 0.275 & 9.77 & 0.154 & 4.22 & 6.48 \\
\hline $6 c$ & & & 1954 & 0.22 & 14.36 & 0.154 & 4.76 & 8.08 \\
\hline $6 \mathrm{~d}$ & & & 770 & 0.22 & 10.53 & 0.154 & 5.204 & 7.636 \\
\hline $6 \mathrm{e}$ & & & 1954 & 0.165 & 15.80 & 0.154 & 6.20 & 9.85 \\
\hline $6 f$ & & & 770 & 0.165 & 11.59 & 0.154 & 6.686 & 9.364 \\
\hline $7 \mathrm{a}$ & \pm 1.0 & 1.0 & 1954 & 0.40 & 11.76 & 0.616 & 24.67 & 35.53 \\
\hline $7 \mathrm{~b}$ & & & 770 & 0.40 & 8.63 & 0.616 & 26.11 & 34.09 \\
\hline $7 \mathrm{c}$ & & & 1954 & 0.32 & 12.67 & 0.616 & 30.27 & 41.97 \\
\hline $7 \mathrm{~d}$ & & & 770 & 0.32 & 9.29 & 0.616 & 31.83 & 40.41 \\
\hline $7 \mathrm{e}$ & & & 1954 & 0.24 & 13.95 & 0.616 & 38.71 & 51.60 \\
\hline $7 \mathrm{f}$ & & & 770 & 0.24 & 10.23 & 0.616 & 40.42 & 4988 \\
\hline $8 \mathrm{a}$ & \pm 1.0 & 2.0 & 1954 & 0.275 & 13.33 & 0.308 & 13.92 & 20.08 \\
\hline $8 \mathrm{~b}$ & & & 770 & 0.275 & 9.77 & 0.462 & 13.61 & 20.39 \\
\hline $8 \mathrm{c}$ & & & 1954 & 0.22 & 14.36 & 0.308 & 17.08 & 23.72 \\
\hline $8 \mathrm{~d}$ & & & 770 & 0.22 & 10.53 & 0.462 & 16.75 & 24.05 \\
\hline $8 \mathrm{e}$ & & & 1954 & 0.165 & 15.80 & 0.462 & 20.03 & 30.97 \\
\hline $8 \mathrm{f}$ & & & 770 & 0.165 & 11.59 & 0.462 & 21.48 & 29.52 \\
\hline
\end{tabular}

In Figure 2 we show the equation of state of the system across the thermal gradient. At low values of the thermal gradient, far from the coexistence curve, we see equations of state that are close to linear. Deviations from linearity seem more prevalent in the sizeasymmetric systems. In addition we note that the decreasing slope of the curves as the dipole magnitude increases, in particular at lower temperature, indicates an increase in the thermal expansivity of the systems.

In Figure 3 we have plotted the reduced heat flux $J^{*}$ computed according to Eqn. 1 across the temperature gradient. Only the component of $\vec{J}$ in the $z$ direction parallel to the thermal gradient is non-zero on average. We find the heat flux to take on a constant value from one end of the thermal gradient to the other. The heat flux increases with the magnitude of the thermal gradient. Size-asymmetric systems have considerably lower heat flux, despite the simulation conditions being identical, i.e. $T / T_{\mathrm{c}}, \rho / \rho_{\mathrm{c}}$, and $\nabla T^{*}$ are the same in many cases. We have noted this trend in our previous work on apolar systems, where we related it to 


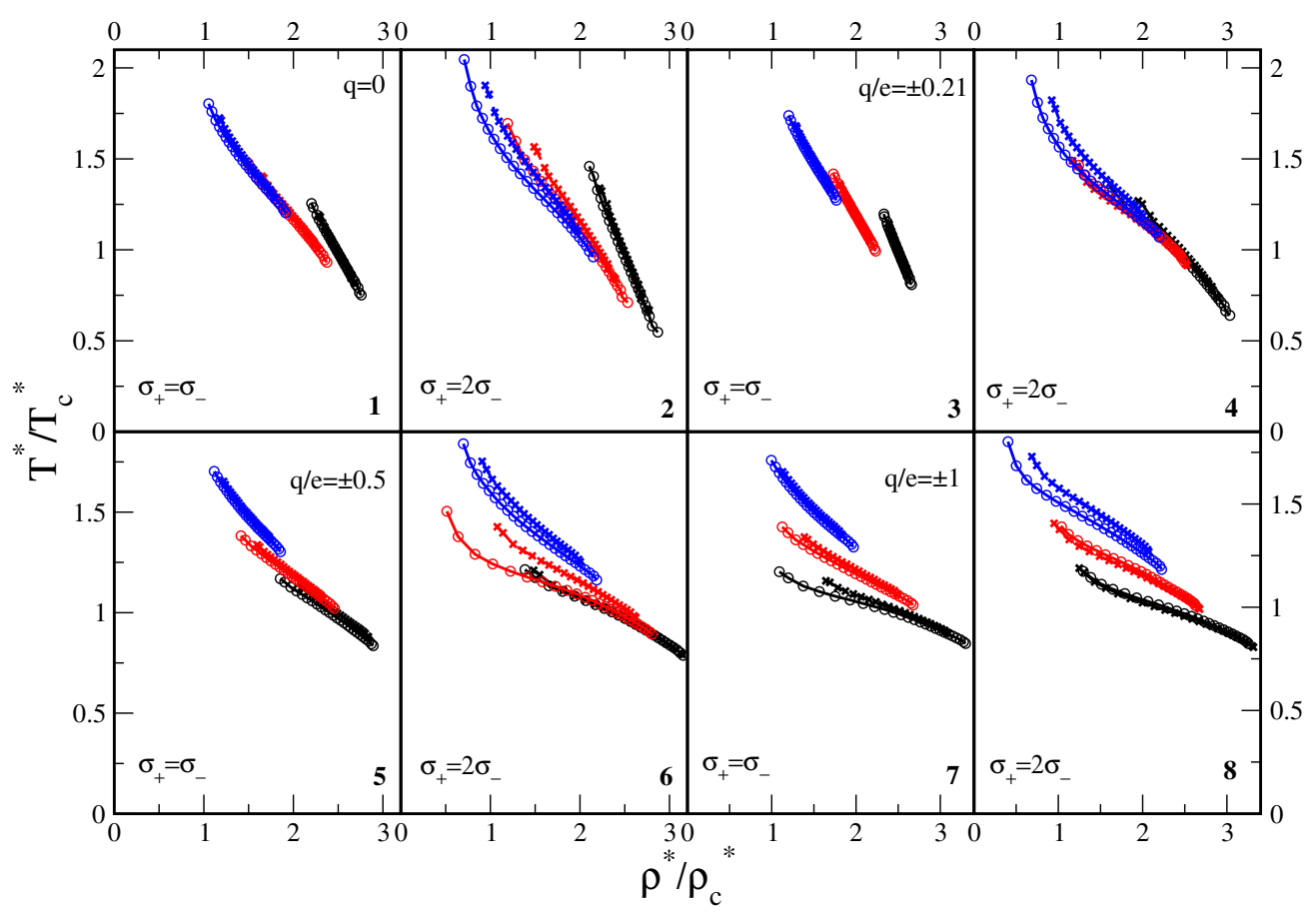

FIG. 2: Plots of the equation of state relating $T^{*}$ and $\rho^{*}$ across the temperature gradient. Numbers in the corners of each graph correspond to the numbers in Table I. Solid lines and circles correspond to the larger system (letters a,c,e in Table I), dashed lines and $\times$ 's are for the smaller system (b,d,f). Line colours are as follows: black, systems a and b, red, systems c and d, and blue, systems e and f. Results were averaged over the two halves of the simulation cell.

the lowering of thermal conductivity in size asymmetric systems [3].

We turn now to the TMO effect. In Figure 4 we show the average cosine of the angle $\theta_{\mu z}$ between the molecular dipole $\vec{\mu}$ and the $z$ axis (parallel to $\nabla T$ ), scaled by the thermal gradient so that different systems can be compared. In the apolar system the direction of the orientation is the same as in our previous study [3], with the larger atom tending to point toward the middle of the simulation box where $T=T_{\text {hot }}$ region, but quantitative results are somewhat different. Sharp peaks in the orientation appear close to the $T_{\text {cold }}$ regions. Closely related apolar dumbbell systems show formation of an amorphous solid phase at similar temperatures [43]. We also observe solidification in our systems at low temperature and this is a likely explanation for these peaks. Another interesting detail is the slope in the plots, showing a trend of less TMO at higher temperatures when the average temperature $T_{\mathrm{av}}=T_{\mathrm{c}}$, but the opposite trend when $T_{\mathrm{av}}=1.2 T_{\mathrm{c}}$.

Our focus in this paper, however, will be the results in the dipolar systems. Here the 


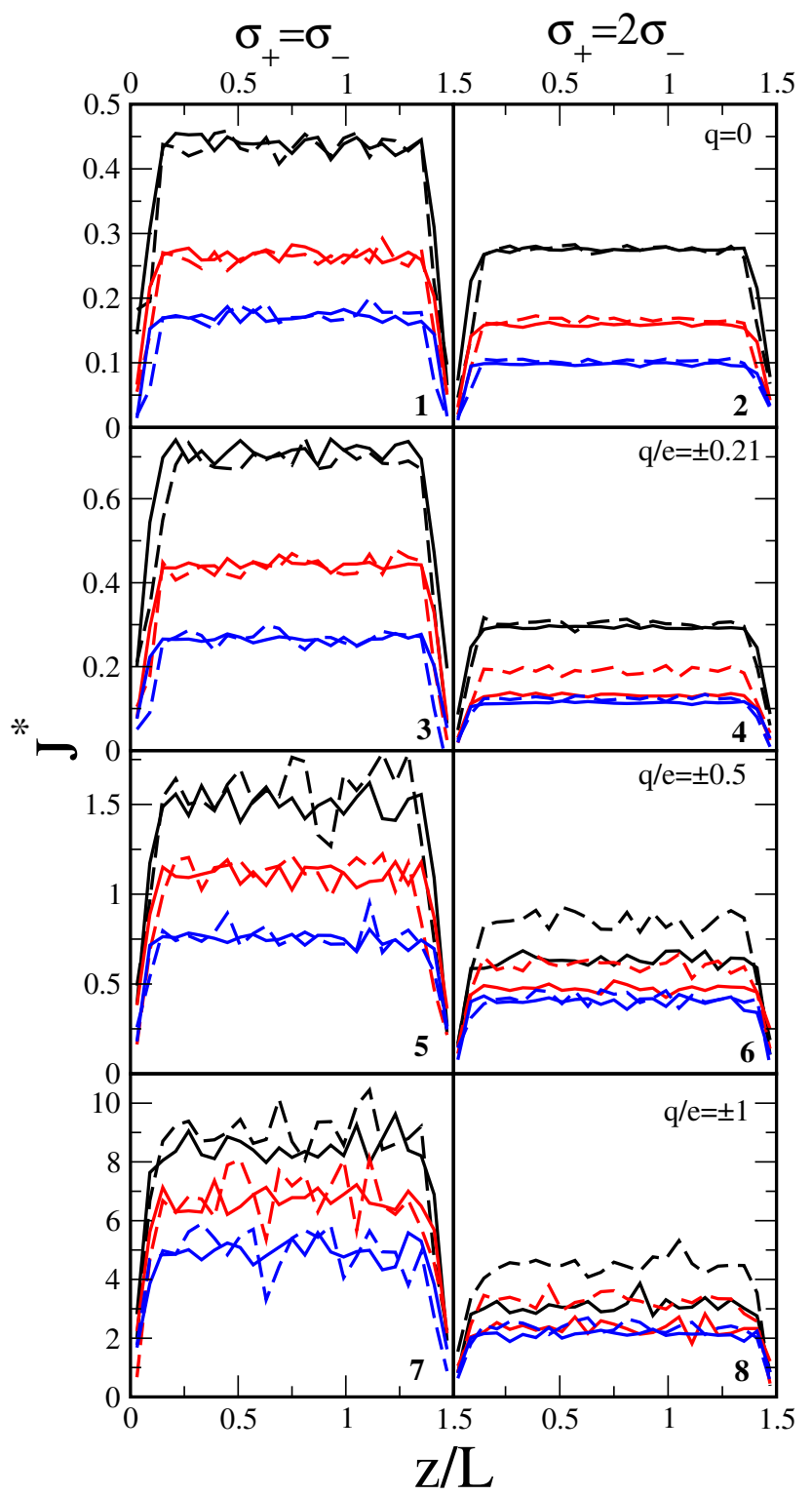

FIG. 3: Plots of the reduced heat flux $J^{*}$ across the simulation box. Numbers in the corners of each graph correspond to the numbers in Table I. Solid lines correspond to the larger system (letters a,c,e in Table I), dashed lines are for the smaller system (b,d,f). The size-symmetric systems are on the left, while the size-asymmetric systems are on the right. Line colours have the same meaning as in Figure 2. Results were averaged over the two halves of the simulation cell.

direction of the orientation remains the same but compared with the apolar systems we see a smooth increase in the TMO from one side of the thermal gradient to the other. In cases where the same gradient magnitude was applied in both system sizes, the shape of the curves are similar but a higher maximum is reached in the larger system. In a sufficiently large 


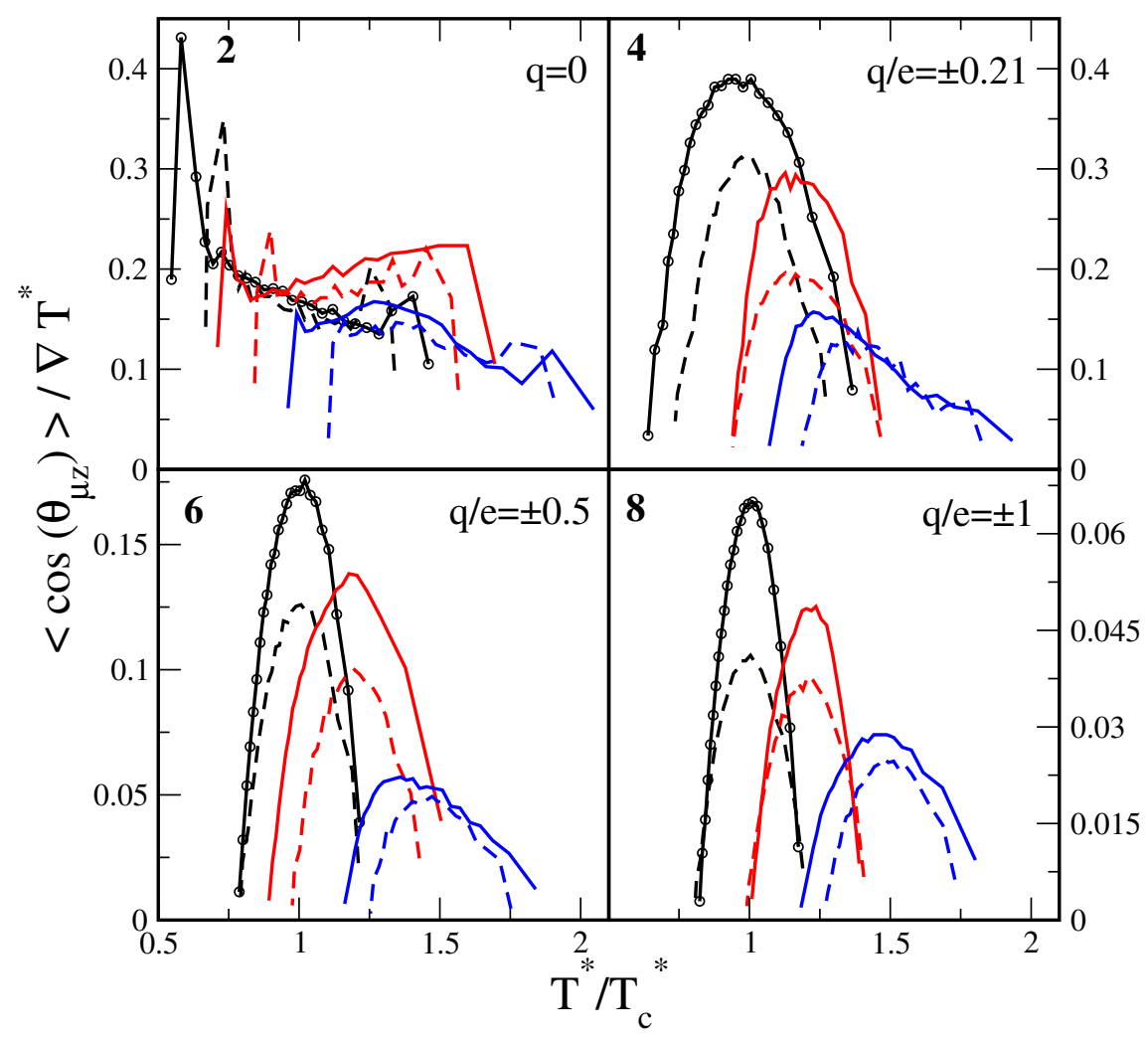

FIG. 4: Plots of the average molecular orientation $\cos \left(\theta_{\mu z}\right)$ scaled by the size of the applied temperature gradient $\nabla T$ as a function of the local temperature. Line colours and styles have the same meanings as in Figure 3. Results were averaged over the two halves of the simulation cell.

system and in a smaller thermal gradient, we should see a gradual increase in the TMO away from the hot and cold regions, and a broad linear increase in the middle of the cell, as seen in both the apolar case and in the studies of TMO in water [4]. Compared with the apolar case, larger correlation lengths for Coulombic interactions mean that much larger systems must be studied in order to reach the linear regime. To confirm that we can reach this regime we have completed simulations of much larger systems in the $q / e= \pm 1$ case, shown in Figure 5. From the viewpoint of experimental verification of TMO it is encouraging that reaching this limit required such large simulations, suggesting that the much smaller overall temperature gradients and larger length scales the experimental setup would be limited to might still result in a significant TMO effect.

The TMO scaled by the thermal gradient is largest in the system with smaller dipoles. Since the induced electric field strength $E_{z}=-P_{z} / \epsilon_{0}$ and the polarization $P_{z}=\rho\langle\vec{\mu}\rangle=$ $\rho|\vec{\mu}|\left\langle\cos \left(\theta_{\mu z}\right)\right\rangle$, we can compute the maximum TMO-induced electric field strength $E_{\max }$ 


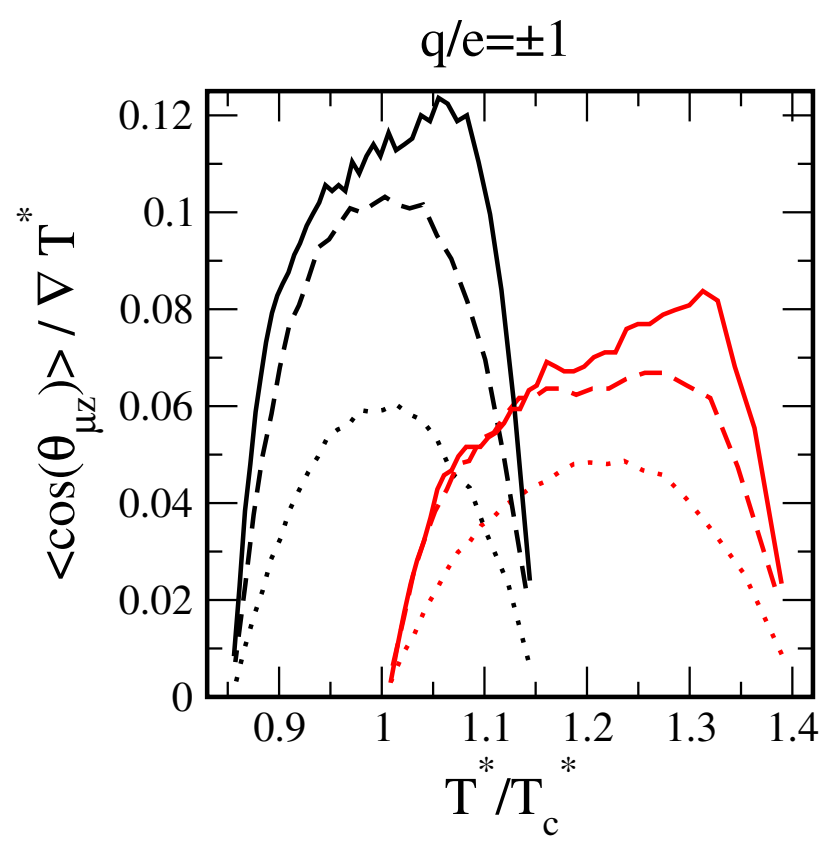

FIG. 5: Plots of the average molecular orientation $\cos \left(\theta_{\mu z}\right)$ scaled by the size of the applied temperature gradient $\nabla T$ as a function of the local temperature, in simulations with the length of the $z$ axis (parallel to the direction of $\nabla T$ ) increased by a factor of two (dashed lines) or three (solid lines), and with the same average density. The dotted lines are for the initial system size of $\mathrm{N}=1954$ particles, i.e. systems $8 \mathrm{a}$ and $8 \mathrm{c}$ in Table I, except the results for system $8 \mathrm{a}$ are in a lower temperature gradient of $\nabla T^{*}=0.25$. The magnitude of the gradient has been adjusted in the larger systems to keep $T_{\text {hot }}$ and $T_{\text {cold }}$ constant.

from the maxima in Figures 4 and 5 and our model parameters and simulation conditions. We find that $E_{\max }$ varies from $2.3 \times 10^{7} \mathrm{~V} / \mathrm{m}$ in system 4 a up to $6.4 \times 10^{7} \mathrm{~V} / \mathrm{m}$ in system 8a. However, the values of $\nabla T^{*}$ in these simulations are considerably different. When we account for this, $E_{\max }$ for a simulation at a given value of $T / T_{\mathrm{c}}$ and $\rho / \rho_{\mathrm{c}}$ is remarkably constant in all of our simulations. We can compare, for example, the largest system with $q / e= \pm 1$ in Figure 5 , in a thermal gradient very close to that of system 4 a we obtain $E_{\max }=$ $2.9 \times 10^{7} \mathrm{~V} / \mathrm{m}$. In summary, regardless of the molecular dipole strength, in a temperature gradient $\nabla T=1 \mathrm{~K} / \AA$ we can expect an induced field strength $|E| \sim 2.5 \times 10^{7} \mathrm{~V} / \mathrm{m}$, perhaps an order of magnitude less than what has been computed for water [5-7].

We also investigated the second order orientation effect by computing the average of the second Legendre polynomial $P_{2}\left[\cos \left(\theta_{\mu z}\right)\right]=\frac{3 \cos ^{2}\left(\theta_{\mu z}\right)-1}{2}$. This function equals 1 when $\vec{\mu}$ is exactly aligned with the gradient direction $z$, but does not distinguish between parallel 


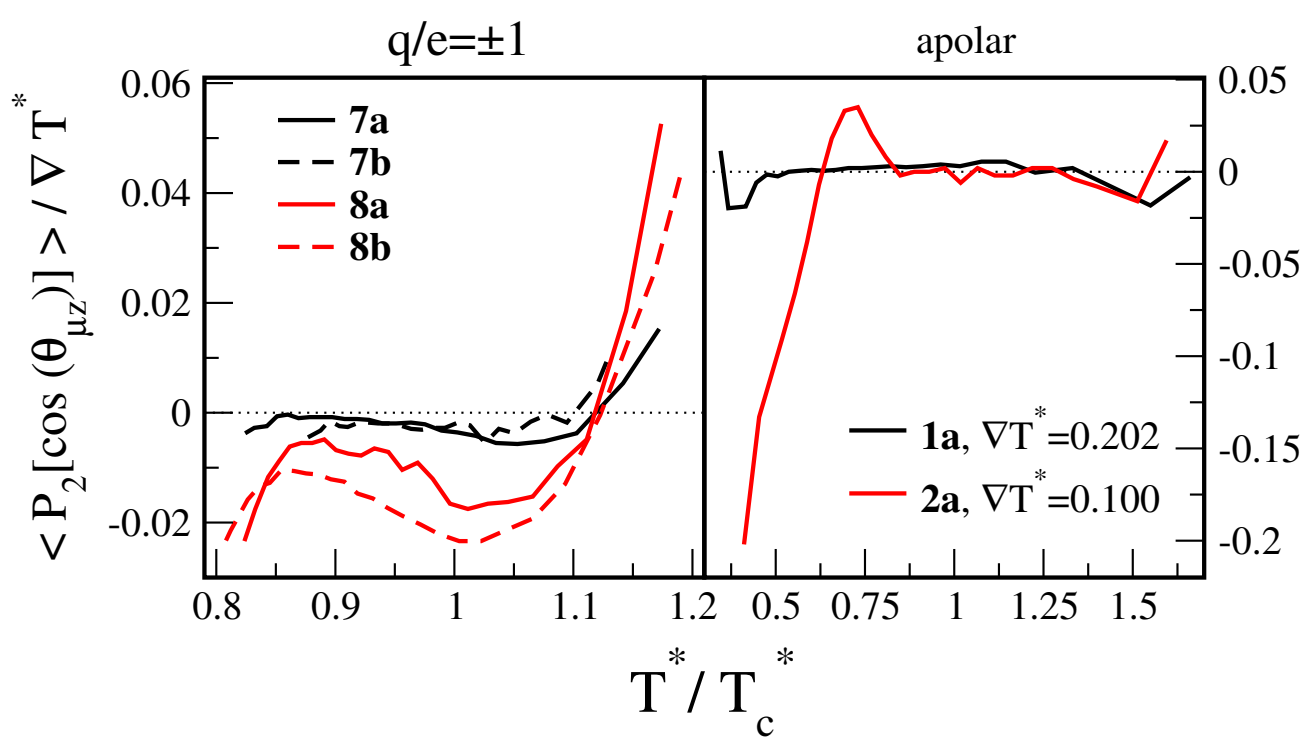

FIG. 6: Plots of the $2^{\text {nd }}$ order Legendre polynomial of the average molecular orientation $P_{2}\left[\cos \left(\theta_{\mu z}\right)\right]$ scaled by the size of the applied temperature gradient $\nabla T^{*}$ as a function of the local temperature. Results were averaged over the two halves of the simulation cell.

or antiparallel directions. Conversely, when the function equals -1 it indicates perfect orientation perpendicular to the direction $z$.

We show these results in Figure 6. In our case, we see that the size-asymmetric systems with large dipoles show a weak second order orientation effect whereby dipolar orientations perpendicular to the $z$ axis are slightly preferred in most of the simulation box, except very near the hot region, where (anti)parallel orientations are preferred. There is no contradiction here between the second order orientation and the first order TMO effect we already described; both of these effects are relatively weak, so it is certainly possible for both of these trends to coexist, and they describe the response to different types of perturbations. What may be most interesting is the observation that even in the size-symmetric case, which of course cannot give rise to a first order TMO effect, we observe a similar (albeit even weaker than in the asymmetric systems) second order orientation. These effects are strongest in the systems with the largest dipoles. There is still a measurable effect in the $q / e= \pm 0.5$ asymmetric system (not shown). We can even detect a weak effect in the apolar systems, but only by increasing the temperature gradient to as large a value as possible without causing liquid-vapour phase separation. As in the first order TMO, the relatively large value of the second order TMO at low temperature is related to the onset of solidification. 
Finally, we present the rotational correlation distribution functions and times. First, we show in Figure 7 both linear and semi-logarithmic plots of the second order rotational correlation functions $C_{2}(t)$ for the size-asymmetric systems computed from Eqn. 2. The apolar system and the $q / e= \pm 0.21$ system may be the most Debye-like, showing a smooth transition to exponential decay after a brief period $(<0.3 \mathrm{ps})$ of ballistic motion. The systems with larger dipoles bear the closest resemblance to models of real polar liquids such as water $[44,45]$, showing a rapid decay due to fast librational motion at timescales less than those for intermolecular bond breaking ( $\sim 1 \mathrm{ps})$ followed by a long period that is well-described by a decaying exponential.

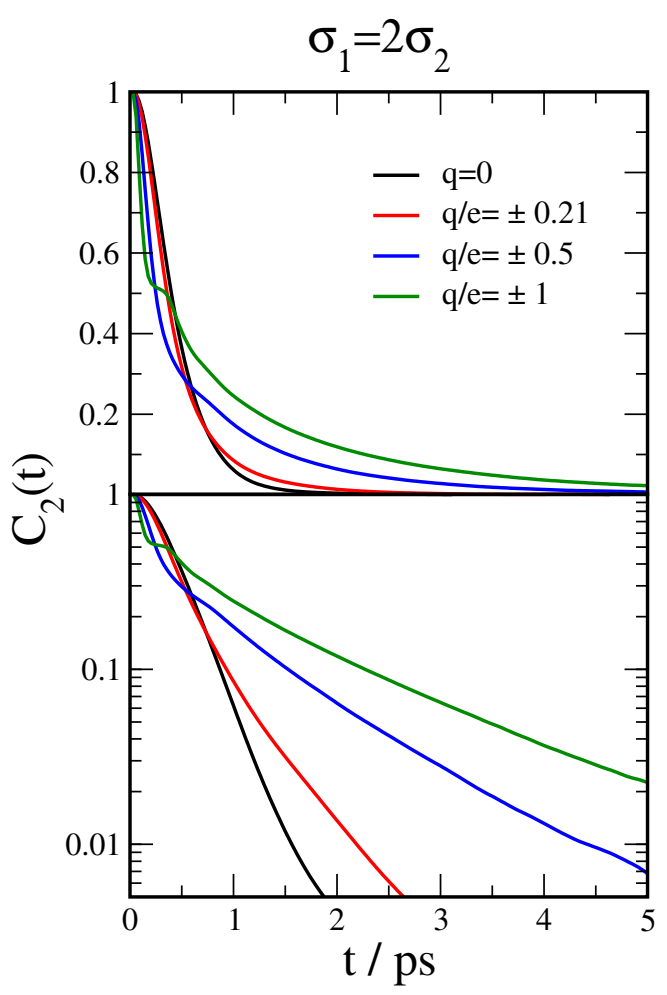

FIG. 7: Plots of the second order rotational correlation function $C_{2}(t)$ obtained from Eqn. 2 for equilibrium simulations with no applied temperature gradient at $T^{*}=T_{c}^{*}, \rho^{*}=2.5 \rho_{c}^{*}$. Top: linear scale. Bottom: logarithmic scale.

In Figure 8 we show the integrated rotational times $\tau_{2}$ computed from Eqn. 3 in simulations with various thermostatting methods for the systems with $q= \pm 1$. To our surprise there was a large effect on the rotational times using a Langevin thermostat, that exists despite the temperatures of all degrees of freedom being well controlled. However, raising the damping time parameter in the thermostat was sufficient to reduce the deviation between 
Langevin thermostat results and results obtained without thermostatting ( $N V E$ ensemble) or with a Nosé-Hoover thermostat. Unfortunately, with a higher damping time we could not adequately control the temperature gradient in the NEMD simulations. Since the effect of the thermostat itself did not extend into the simulation cell outside the hot and cold regions, we chose to accept that the rotational times would be wrong in these regions but not in the rest of the simulation box.

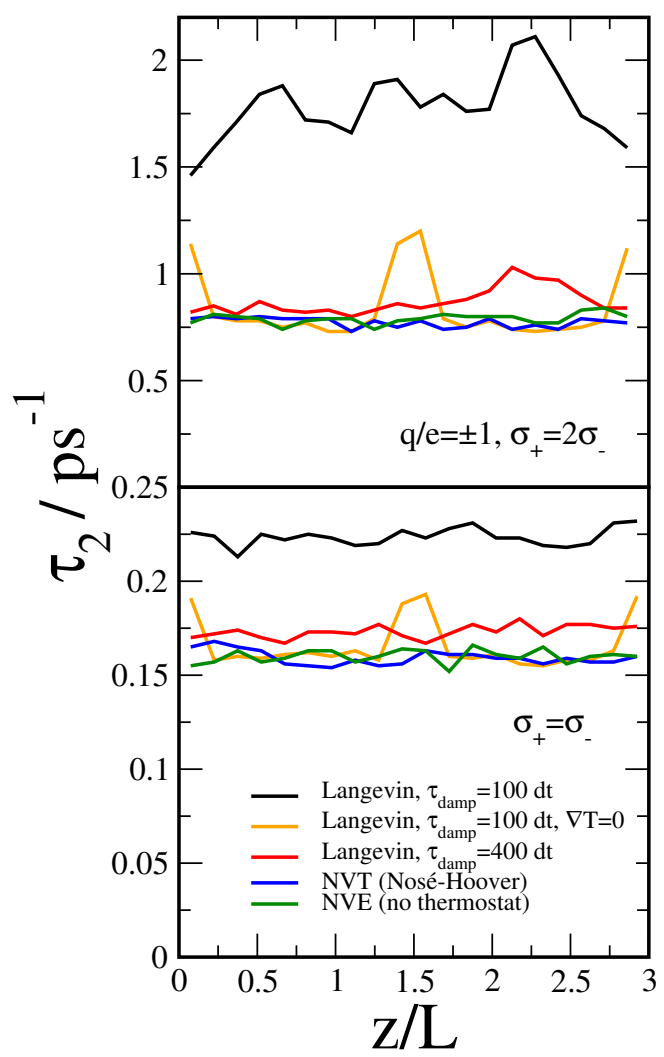

FIG. 8: Plots of the second order rotational correlation time $\tau_{2}$ obtained from Eqn. 3 as a function of the $z$ coordinate across the simulation box for different thermostatting methods, all with $T^{*}=$ $T_{\mathrm{c}}^{*}, \rho^{*}=2.5 \rho_{\mathrm{c}}^{*}$. The line with $\nabla T=0$ is a simulation done as if a thermal gradient were applied as described for the NEMD simulations, but with $T_{\text {cold }}=T_{\text {hot }}=T_{\mathrm{c}}$.

In Figure 9 we show for the systems with $q= \pm 1$ the integrated rotational times computed across the temperature gradients for three of the NEMD conditions; $T_{\mathrm{av}}=T_{\mathrm{c}}$ and $\rho_{\mathrm{av}}=2.5 \rho_{\mathrm{c}}$ for both large and small systems, and $T_{\mathrm{av}}=1.2 T_{\mathrm{c}}, \rho_{\mathrm{av}}=2 \rho_{\mathrm{c}}$ for the larger system only. These results are plotted against both the temperature and density obtained from the equations of state (Figure 2). We also show for comparison results from equilibrium simulations in the NVT ensemble at state points taken from the same equations of state. 


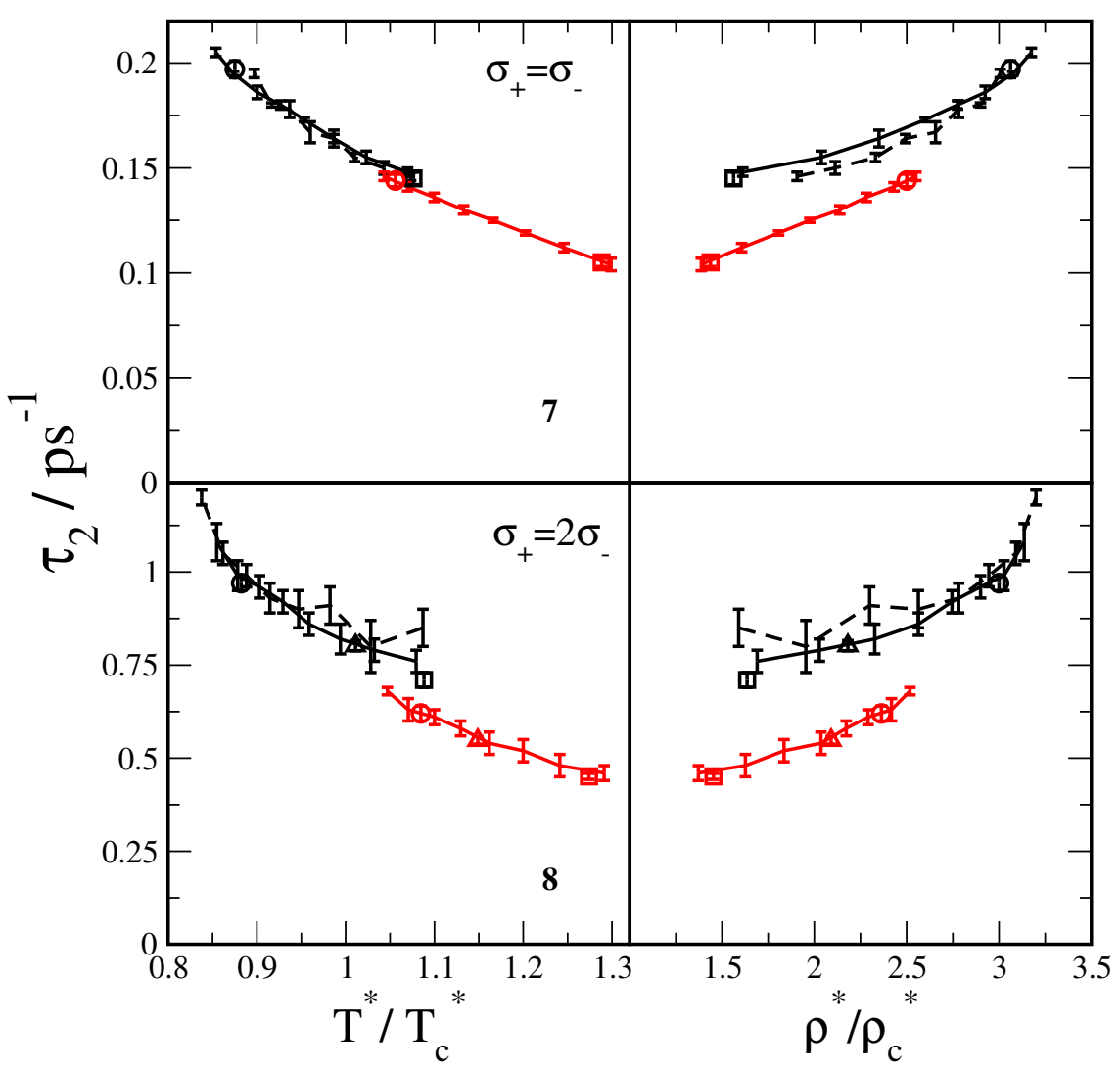

FIG. 9: Plots of the second order rotational correlation time $\tau_{2}$ obtained from Eqn. 3 as a function of the local temperature $T^{*}$ (left) or local density $\rho^{*}$ (right) across the simulation box for both NEMD simulations (lines) and from canonical ensemble simulations at different state points along the equations of state (open symbols). Line colours and styles are as in Figure 3.

Considering each curve separately, the trends are as expected; as temperature increases along each equation of state, the rotational time decreases, and as the density increases, the rotational time increases. However, when comparing across the different NEMD simulations some surprising results are revealed. For instance, even though two of the equilibrium simulations in the size-asymmetric system have virtually the same $T^{*} \sim 18.5$ (shown by the black square and the red circle in the lower half of Figure 9), it is the simulation at the higher density $\rho^{*}=0.26$ which has a significantly faster rotational time than the simulation with $\rho^{*}=0.18$.

Although these results are interesting, our main reason for computing the rotational correlation times was to determine if we could detect any influence from the thermal gradient. However, it is clear from Figure 9 that there is no significant difference in the rotational 
times obtained from the NEMD simulations versus the equilibrium simulations.

\section{CONCLUSIONS}

We have completed a thorough MD simulation study of dipolar dumbbells. We have computed their liquid-vapour critical points and studied the behaviour of these systems in thermal gradients. The NEMD simulations provide new data on equations of state and heat transfer properties of these systems over a range of thermodynamic states. Interestingly, size asymmetric dumbbells have significantly lower heat flux given the same thermal gradient. We have measured the thermomolecular orientation (TMO) effect in these systems. Our findings indicate that the largest TMO occurs at temperatures as near as possible to the critical temperature. The TMO-induced electric field, when scaled by the size of the thermal gradient, is not strongly affected by the magnitude of the molecular dipole moment. This might indicate that the best candidate systems for experimental observation of TMO should not be those with the largest molecular dipoles, but could depend on other factors, such as liquid phase stability over a large temperature range suitable for applying as large a thermal gradient as possible.

We have also noted a novel second order TMO, where a size-symmetric dipolar system in a thermal gradient shows a weak preference for orientations perpendicular to the gradient at low temperatures and in the middle of the simulation box, but a preference for (anti-)parallel orientations near a source of heat. The only asymmetry in this system is the dipole moment, which should lead to a TMO arising from the minimization of the entropy production $[2,4]$. Even in the apolar size-symmetric system, we observe a measurable second order TMO in a very large thermal gradient. Part of the difficulty in understanding these new effects is that we cannot effectively separate the contributions of the size or shape asymmetry from the effect of the electric dipole, since they point in parallel directions. It would be interesting to examine TMO effects in a polar liquid with a mass or size asymmetry in a different direction from the molecular dipole, eg. HDO (i.e. singly deuterated water).

Finally, we have computed rotational correlation functions in these systems, as a step towards modelling possible spectroscopic signatures of TMO in real polar fluids such as water or acetonitrile. Our attempted analysis based on integrating the correlation functions

to obtain a correlation time revealed no difference between the equilibrium systems and the 
systems in a thermal gradient. Further progress will require us to more carefully consider how to correctly model the molecular rotational dynamics in a thermal gradient. For example, we have not dealt with the obvious fact that the imposed orientational anisotropy ought to lead to anisotropy in the rotational dynamics as well. A more involved simulation approach would include molecular electronic polarizability as well as collective effects, similar to what has been used with some success to describe effects of confinement on the OKE spectrum of acetonitrile[38]. Another approach would be to compute the vibrational density of states via Fourier transforms of the velocity autocorrelation functions[46] and attempt to relate these to the spectroscopic response.

As an aside, we have noted some unexpected effects arising from different thermostatting methods. It appears that the Nosé-Hoover thermostat as well as velocity scaling have some difficulty with properly equilibrating stiff yet flexible bonds, at least in the implementations of these thermostats we have used. Using chained thermostats might be one way of improving this equilibration without abandoning the Nosé-Hoover thermostat entirely [47]. The Langevin thermostat is more effective at equilibrating these vibrations but on the other hand, gives significantly slower rotational dynamics. We intend to carefully examine all of these technical details in further work.

\section{Acknowledgments}

We thank Ulf Østerberg for many helpful discussions. We thank The Research Council of Norway (Project 221675) and the EPSRC (EP/J003859/1) for financial support, and NOTUR for computational resources. F.B. acknowledges the award of an EPSRC Leadership Fellowship.

[1] S. Wiegand, J. Phys. Condens. Matter 16, R357 (2004).

[2] F. Römer, F. Bresme, J. Muscatello, D. Bedeaux, and J. M. Rubi, Phys. Rev. Lett. 108, 105901 (2012).

[3] F. Römer and F. Bresme, Mol. Simul. 38, 1198 (2013).

[4] F. Bresme, A. Lervik, D. Bedeaux, and S. Kjelstrup, Phys. Rev. Lett. 101, 020602 (2008).

[5] J. Muscatello, F. Römer, J. Sala, and F. Bresme, Phys. Chem. Chem. Phys. 13, 19970 (2011). 
[6] J. A. Armstrong and F. Bresme, J. Chem. Phys. 139, 014504 (2013).

[7] J. A. Armstrong, A. Lervik, and F. Bresme, J. Phys. Chem. B 117, 14817 (2013).

[8] F. Römer, Z. Wang, S. Wiegand, and F. Bresme, J. Phys. Chem. B 117, 8209 (2013).

[9] K. S. Pitzer, Acc. Chem. Res. 23, 333 (1990).

[10] P. J. Camp and G. N. Patey, J. Chem. Phys. 111, 9000 (1999).

[11] F. Bresme, E. Lomba, J. J. Weis, and J. L. F. Abascal, Phys. Rev. E 51, 289 (1995).

[12] J. N. Aqua, S. Banerjee, and M. E. Fisher, Phys. Rev. E 72, 041501 (2005).

[13] C. D. Daub, G. N. Patey, and P. J. Camp, J. Chem. Phys. 119, 7952 (2003).

[14] G. Ganzenmüller and P. J. Camp, J. Chem. Phys. 126, 191104 (2007).

[15] H. Braun and R. Hentschke, Phys. Rev. E 80, 041501 (2009).

[16] H. Braun and R. Hentschke, Phys. Rev. E 87, 012311 (2013).

[17] S. Dussi, L. Rovigatti, and F. Sciortino, Mol. Phys. 111, 3608 (2013).

[18] V. Talanquer and D. W. Oxtoby, J. Chem. Phys. 103, 3686 (1995).

[19] J. M. Romero-Enrique, G. Orkoulas, A. Z. Panagiotopoulos, and M. E. Fisher, Phys. Rev. Lett. 85, 4558 (2000).

[20] Q. Yan and J. J. de Pablo, J. Chem. Phys. 114, 1727 (2001).

[21] Q. Yan and J. J. de Pablo, Phys. Rev. Lett. 86, 2054 (2001).

[22] Q. Yan and J. J. de Pablo, J. Chem. Phys. 116, 2967 (2002).

[23] H. V. Spohr and G. N. Patey, J. Chem. Phys. 129, 064517 (2008).

[24] M. Bohn, R. Lustig, and J. Fischer, Fluid Phase Equilib. 25, 251 (1986).

[25] S. J. Plimpton, J. Comp. Phys. 117, 1 (1995).

[26] D. Wolf, P. Keblinski, S. R. Phillpot, and J. Eggebrecht, J. Chem. Phys. 110, 8254 (1999).

[27] C. J. Fennell and J. D. Gezelter, J. Chem. Phys. 124, 234104 (2006).

[28] J. Muscatello and F. Bresme, J. Chem. Phys. 135, 234111 (2011).

[29] J. P. Ryckaert, G. Ciccotti, and H. J. C. Berendsen, J. Comp. Phys. 23, 327 (1977).

[30] T. Kraska, F. Römer, and A. R. Imre, J. Phys. Chem. B 113, 4688 (2009).

[31] N. B. Wilding and A. D. Bruce, J. Phys.: Condens. Matter 4, 3087 (1992).

[32] T. Schneider and E. Stoll, Phys. Rev. B 17, 1302 (1978).

[33] F. Bresme and J. Armstrong, J. Chem. Phys. 140, 016102 (2014).

[34] T. W. Sirk, S. Moore, and E. F. Brown, J. Chem. Phys. 138, 064505 (2013).

[35] B. J. Berne and G. D. Harp, Adv. Chem. Phys. 17, 63 (1970). 
[36] A. R. Bizzarri and S. Cannistraro, J. Phys. Chem. B 106, 6617 (2002).

[37] A. Y. Zasetsky, S. V. Petelina, A. K. Lyashchenko, and A. S. Lileev, J. Chem. Phys. 133, 134502 (2010).

[38] A. A. Milischuk and B. M. Ladanyi, J. Phys. Chem. B 117, 15729 (2013).

[39] L. Hua, X. H. Huang, R. H. Zhou, and B. J. Berne, J. Phys. Chem. B 110, 3704 (2006).

[40] I. Benjamin, J. Phys. Chem. B 2005, 13711 (2005).

[41] S. Nosé, Mol. Phys. 52, 255 (1984).

[42] W. G. Hoover, Phys. Rev. A 31, 1695 (1985).

[43] C. Vega, C. McBride, E. de Miguel, F. J. Blas, and A. Galindo, J. Chem. Phys. 118, 10696 (2003).

[44] D. van der Spoel, P. J. van Maaren, and H. J. C. Berendsen, J. Chem. Phys. 108, 10220 (1998).

[45] F. Bresme, B. Hafskjold, and I. Wold, J. Phys. Chem. 100, 1879 (1996).

[46] R. M. Lynden-Bell, L. Xue, G. Tamas, and E. L. Quitevis, J. Chem. Phys. 141, 044506 (2014).

[47] G. J. Martyna, M. L. Klein, and M. Tuckerman, J. Chem. Phys. 97, 2635 (1992). 